\title{
Optimality conditions for an exhausterable function on an exhausterable set
}

\author{
Majid E. Abbasov \\ St. Petersburg State University, SPbSU, \\ 7/9 Universitetskaya nab., St. Petersburg, 199034 Russia. \\ m.abbasov@spbu.ru,abbasov.majid@gmail.com
}

February 26, 2019

\begin{abstract}
Exhausters are families of convex compact sets that allow one to represent directional derivative of the studied function at the considered point in the form of InfMax or SupMin of linear functions. Functions for which such a representation is valid we call exhausterable. The class of these functions is quite wide and contains many nonsmooth ones. The set which is given by exhausterable function is also called exhausterable.

In the present paper we describe optimality conditions for an exhausterable function on an exhausterable set. These conditions can be used for solving of many nondifferentiable optimization problems. An example that illustrate obtained results is provided.

Keywords: Exhausters, Nonsmooth analysis, Nondifferentiable optimization, Constrained optimization, Optimality conditions
\end{abstract}

\section{Introduction}

Directionally differentiable functions form a wide and important class of nonsmooth functions. It includes convex functions, maximum and minimum functions and others.

Subdifferential notion [1] can be used effectively for working with directional derivative and therefore for solution of convex nonsmoth optimization problems. Researchers tried to develop approaches also for nonconvex problems. The most known invented tools are subdifferentials of Clarke [2, 3, Mordukhovich [4, Michel-Penot [5,6] and others. The concept of exhausters holds a special place in this series due to its constructiveness.

The idea of exhausters notion goes back to the works of Pshenichny, Rubinov and Demyanov. Pshenichny in [7] introduced the definition of upper convex approximation. Demyanov and Rubinov [8,9] proposed to consider exhaustive families of upper convex and lower concave approximations, and then introduced exhauster notion [10,11.

Exhausters are families of convex compact sets that allow one to represent the directional derivative of the studied function in the form of InfMax or SupMin of linear functions. Therefore they provide the same representation for the 
approximation of a studied function in the neighbourhood of a considered point. Functions for which such an expansion is valid we call exhausterable.

Calculus of exhausters was described in 11]. Formulas of this calculus allows one to build exhausters for a wide class of functions. Unconstrained optimality conditions in terms of these families were derived in [11 14. Constrained optimality conditions for an exhausterable function on an abstractive theoretical cone were obtained in [15].

In the present work we study constrained optimality conditions for exhausterable function on a set which is defined via another exhausterable function. We get new optimality conditions in terms of exhausters of these two functions.

The paper is organized as follows. In Section 1 we discuss directional derivative and exhausters notions. In Section 2 we consider the statement of the problem and conic approximations of a feasible set. Optimality conditions for an exhausterable function on an exhausterable set are given in Section 3. An illustrative example is provided in Section 4.

\section{Dini and Hadamard directional derivatives. Exhausters}

Let a function $f: \mathbb{R}^{n} \rightarrow \mathbb{R}$ be given. The function $f$ is called Dini-differentiable at a point $x \in \mathbb{R}^{n}$ in a direction $g \in \mathbb{R}^{n}$, if there exists the finite limit

$$
f_{D}^{\prime}(x, g)=\lim _{\alpha \downarrow 0} \frac{f(x+\alpha g)-f(x)}{\alpha} .
$$

The function $f$ is called Hadamard-differentiable at a point $x \in \mathbb{R}^{n}$ in a direction $g \in \mathbb{R}^{n}$, if there exists the finite limit

$$
f_{H}^{\prime}(x, g)=\lim _{\left[\alpha, g^{\prime}\right] \rightarrow[+0, g]} \frac{f\left(x+\alpha g^{\prime}\right)-f(x)}{\alpha} .
$$

The value $f_{D}^{\prime}(x, g)$ is called the Dini derivative of the function $f$ at the point $x \in \mathbb{R}^{n}$ in the direction $g \in \mathbb{R}^{n}$, and the value $f_{H}^{\prime}(x, g)$ is called the Hadamard derivative of the function $f$ at the point $x \in \mathbb{R}^{n}$ in the direction $g \in \mathbb{R}^{n}$. The functions $f_{D}^{\prime}(x, g)$ and $f_{H}^{\prime}(x, g)$ are positively homogeneous (p.h.) as functions of direction $g \in \mathbb{R}^{n}$. It is clear that differentiability in the sense of Hadamard implies differentiability in the sense of Dini. The converse is not true.

Let $f: \mathbb{R}^{n} \rightarrow \mathbb{R}$ be a directionally differentiable (in the sense of Dini or Hadamard) function and $h(g)=f^{\prime}(x, g)$ be the corresponding derivative of the function $f$ at a point $x$ in a direction $g$. Fix $x \in \mathbb{R}^{n}$. In case when $h(g)$ is upper semicontinuous as a function of $g$ it can be written in the form (see [8])

$$
h(g)=\inf _{C \in E^{*}} \max _{v \in C}\langle v, g\rangle,
$$

where $E^{*}=E^{*}(x)$ is a family of convex, closed and bounded sets from $\mathbb{R}^{n}$.

If $h(g)=f^{\prime}(x, g)$ is lower semicontinuous as a function of $g$, then it can be expressed as

$$
h(g)=\sup _{C \in E_{*}} \min _{w \in C}\langle w, g\rangle,
$$


where $E_{*}=E_{*}(x)$ is a family of convex, closed and bounded sets from $\mathbb{R}^{n}$.

The family of sets $E^{*}$ is called an upper exhauster of the function $f$ at the point $x$ (respectively, in the sense of Dini or Hadamard), while the family $E_{*}$ is called a lower exhauster of the function $f$ at the point $x$ (respectively, in the sense of Dini or Hadamard).

It is obvious that an exhauster of the function $f$ at the point $x$ is also the exhauster of the function $h(g)$ at the origin.

In case when $h$ is continuous at $g$, then the both representations (11) and (2) are true. In [16] it was shown that if $h$ is Lipschitz then this function can be written both in forms

$$
h(g)=h_{1}(g)=\min _{C \in E^{*}} \max _{v \in C}\langle v, g\rangle \quad \forall g \in \mathbb{R}^{n},
$$

and

$$
h(g)=h_{2}(g)=\max _{C \in E_{*}} \min _{w \in C}\langle w, g\rangle \quad \forall g \in \mathbb{R}^{n},
$$

where the families of sets $E^{*}$ and $E_{*}$ are totally bounded. Recall that a family of sets $E$ is totally bounded if there exists a ball $B$ in $\mathbb{R}^{n}$ such that

$$
C \subset B \quad \forall C \in E .
$$

The functions $h_{1}$ and $h_{2}$ give p.h. approximations of the increment of the function $f$ in a neighborhood of the point $x$. In what follows we will deal with representations (3) and (4).

Exhausters were introduced in [10,11. This notion brought attention of many researchers 19 24. It turned out that unconstrained optimality conditions for the minimum most organically can be expressed in terms of upper exhausters (see 11,17,18]). Therefore an upper exhauster was called proper for the minimization problem and adjoint for the maximization one.

Theorem 1. If a function $f(x)$ attains a local minimum at a point $x_{*}$ and an upper exhauster $E^{*}$ (in the sense of Dini or Hadamard) of the function $f(x)$ at the point $x_{*}$ is known, then

$$
h(g)=f^{\prime}\left(x_{*}, g\right)=\min _{C \in E^{*}} \max _{v \in C}\langle v, g\rangle \geq 0 \quad \forall g \in \mathbb{R}^{n},
$$

what is equivalent to the condition

$$
0_{n} \in C \quad \forall C \in E^{*} .
$$

Theorem 2. If a function $f(x)$ attains a local maximum at a point $x^{*}$ and an upper exhauster $E^{*}$ (in the sense of Dini or Hadamard) of the function $f(x)$ at the point $x^{*}$ is known, then

$$
h(g)=f^{\prime}\left(x^{*}, g\right)=\min _{C \in E^{*}} \max _{v \in C}\langle v, g\rangle \leq 0 \quad \forall g \in \mathbb{R}^{n},
$$

what is equivalent to the condition that for every $g \in \mathbb{R}^{n}$ there exists a set $C(g) \in E^{*}$ such that

$$
\langle v, g\rangle \geq 0 \quad \forall v \in C(g) .
$$

Symmetric is the situation with a lower exhauster. This family was called proper for the maximization problem and adjoint for the minimization one. 
Theorem 3. If a function $f(x)$ attains a local maximum at a point $x^{*}$ and a lower exhauster $E_{*}$ (in the sense of Dini or Hadamard) of the function $f(x)$ at the point $x^{*}$ is known, then

$$
h(g)=f^{\prime}\left(x^{*}, g\right)=\max _{C \in E_{*}} \min _{v \in C}\langle v, g\rangle \leq 0 \quad \forall g \in \mathbb{R}^{n},
$$

is equivalent to the condition

$$
0_{n} \in C \quad \forall C \in E_{*} .
$$

Theorem 4. If a function $f(x)$ attains a local minimum at a point $x_{*}$ and a lower exhauster $E_{*}$ (in the sense of Dini or Hadamard) of the function $f(x)$ at the point $x_{*}$ is known, then

$$
h(g)=f^{\prime}\left(x_{*}, g\right)=\max _{C \in E_{*}} \min _{v \in C}\langle v, g\rangle \geq 0 \quad \forall g \in \mathbb{R}^{n},
$$

what is equivalent to the condition that for every $g \in \mathbb{R}^{n}$ there exists a set $C(g) \in E_{*}$ such that

$$
\langle v, g\rangle \geq 0 \quad \forall v \in C(g)
$$

\section{Problem statement. Conic approximations}

Let functions $f: \mathbb{R}^{n} \rightarrow \mathbb{R}$ and $u: \mathbb{R}^{n} \rightarrow \mathbb{R}$ be directionally differentiable in the sense of Hadamard. Consider the problem

$$
\left\{\begin{array}{l}
f(x) \rightarrow \min \\
x \in \Omega
\end{array}\right.
$$

where $\Omega=\left\{x \in \mathbb{R}^{n} \mid u(x) \leq 0\right\}$. Since $f$ and $u$ are Hadamard-differentiable both representations (3) and (4) are valid (see Theorems 5.1 and 3.2 in [8]) for the derivatives $f_{H}^{\prime}(x, g)$ and $u_{H}^{\prime}(x, g)$ at the studied point $x$.

We need conic approximation of the set $\Omega$ in the neighborhood of the studied point $x$ to derive optimality conditions for the problem (7). Remind some definitions.

$K_{p}(x)$ is called cone of possible directions with respect to the set $\Omega$ at point $x$ if for any $y \in K_{p}(x)$ there exists $\bar{\theta}>0$ such that $x+\theta y \in \Omega$ for all $\theta \in[0, \bar{\theta}]$.

$K_{a d}(x)$ is called cone of admissible directions (or Bouligand cone) with respect to the set $\Omega$ at the point $x$ if for any $y \in K_{a d}(x)$ there exists $\left[\theta_{k}, y_{k}\right] \rightarrow$ $[+0, y]$, where $\theta_{k} \geq 0$, such that $x+\theta_{k} y_{k} \in \Omega$.

Also define cones

$$
K_{<}(x)=\left\{y \in \mathbb{R}^{n} \mid u_{H}^{\prime}(x, g)<0\right\}, \quad K_{\leq}(x)=\left\{y \in \mathbb{R}^{n} \mid u_{H}^{\prime}(x, g) \leq 0\right\} .
$$

Since the derivative $u_{H}^{\prime}(x, g)$ is continuous as a function of direction [8], the cone $K_{<}(x)$ is open (if it is not empty) and the cone $K_{\leq}(x)$ is closed.

It can be checked easily that

$$
K_{<}(x) \subset K_{p}(x) \subset K_{a d}(x) \subset K_{\leq}(x) .
$$

We say that the regularity condition holds at the point $x$ if

$$
\operatorname{cl}\left\{K_{<}(x)\right\}=K_{\leq}(x) .
$$

where $\operatorname{cl}\left\{K_{<}(x)\right\}$ is the closure of the cone $K_{<}(x)$. This condition provides constructive way for building the cone $K_{a d}(x)$ which is used in optimal conditions. 


\section{Optimality conditions}

We will need the following results (see $[8]$ ).

Theorem 5. Let $f$ be be directionally differentiable in the sense of Hadamard at the point $x_{*} \in \Omega$. For the point $x_{*}$ to be a local minimizer of $f$ on $\Omega$, it is necessary that

$$
f_{H}^{\prime}\left(x_{*}, g\right) \geq 0 \quad \forall g \in K_{a d}\left(x_{*}\right),
$$

where $K_{a d}\left(x_{*}\right)$ is the Bouligand cone to the set $\Omega$ at the point $x_{*}$.

Theorem 6. Let $f$ be be directionally differentiable in the sense of Hadamard at the point $x^{*} \in \Omega$. For the point $x^{*}$ to be a local maximizer of $f$ on $\Omega$, it is necessary that

$$
f_{H}^{\prime}\left(x^{*}, g\right) \leq 0 \quad \forall g \in K_{a d}\left(x^{*}\right),
$$

where $K_{a d}\left(x^{*}\right)$ is the Bouligand cone to the set $\Omega$ at the point $x^{*}$.

Now we can formulate necessary optimality conditions for the problem (77). We consider in detail only conditions for the minimum, since conditions for the maximum can be derived similarly.

First state minimum conditions in terms of (proper) upper exhauster of the function $f$.

Theorem 7. Let the regularity condition holds at the point $x_{*} \in \Omega$, families of sets $E^{*}(f)$ and $E_{*}(u)$ be an upper and a lower exhausters in the sense of Hadamard of the functions $f$ and $u$ at the point $x_{*}$ respectively. Then for the point $x_{*}$ to be a local minimum of the function $f$ on the set $\Omega$ it is necessary that

$$
\bigcap_{C \in E_{*}(u)} \operatorname{cl}\left\{\mathbb{R}^{n} \backslash K^{+}(C)\right\} \subset \bigcap_{C \in E^{*}(f)} \operatorname{cl}\left\{\mathbb{R}^{n} \backslash\left(-K^{+}(C)\right)\right\},
$$

where $K(C)=$ cone $\{C\}$ is the conic hull of set $C, K^{+}(C)$ is the conjugate cone of $K(C)$.

Proof. Due to Theorem 5 if point $x_{*}$ is a local minimum of the function $f$ on $\Omega$, then

$$
f_{H}^{\prime}\left(x_{*}, g\right) \geq 0 \quad \forall g \in K_{a d}\left(x_{*}\right) .
$$

Whence considering regularity condition we get

$$
f_{H}^{\prime}\left(x_{*}, g\right) \geq 0 \quad \forall g \in K_{\leq}\left(x_{*}\right) .
$$

Using exhauster representation for the directional derivatives in (11) we obtain that inequality

$$
\min _{C \in E^{*}(f)} \max _{v \in C}\langle v, g\rangle \geq 0
$$

holds for any $g$ such that

$$
\max _{C \in E_{*}(u)} \min _{v \in C}\langle v, g\rangle \leq 0 .
$$

This means that for any $g \in \mathbb{R}^{n}$ such that

$$
\min _{v \in C}\langle v, g\rangle \leq 0 \quad \forall C \in E_{*}(u)
$$


holds the condition

$$
\max _{v \in C}\langle v, g\rangle \geq 0 \quad \forall C \in E^{*}(f) .
$$

Inequality (12) is equivalent to the fact that for every $C \in E_{*}(u)$ there exists $v(C) \in C$ such that $\langle v(C), g\rangle \leq 0$. Consequently $g$ does not lie in the interior of the set (cone $\{C\})^{+}$for all $C \in E_{*}(u)$. Thus denoting $K^{+}(C)$ a conjugate cone of cone $\{C\}$ we have $g \in \bigcap_{C \in E_{*}(u)} \operatorname{cl}\left\{\mathbb{R}^{n} \backslash K^{+}(C)\right\}$.

The same way we can show that any $g$ satisfying inequality (13) belongs to the set $\bigcap_{C \in E^{*}(f)} \operatorname{cl}\left\{\mathbb{R}^{n} \backslash\left(-K^{+}(C)\right)\right\}$ and vice versa.

Therefore (12) and (13) implies (10).

Corollary 7.1. Condition (10) can be interpreted as follows: for any hyperplane passing through the origin which nonpositive half-space contains an element from $C \in E_{*}(u)$ for all $C \in E_{*}(u)$, there exists an element from $\widetilde{C}$ which lies in the nonnegative half-space of this hyperplane for all $\widetilde{C} \in E^{*}(f)$.

Theorem 8. Let the regularity condition holds at the point $x_{*} \in \Omega$, families of sets $E^{*}(f)$ and $E^{*}(u)$ be upper exhausters in the sense of Hadamard of the functions $f$ and $u$ at the point $x_{*}$ respectively. Then for the point $x_{*}$ to be a local minimum of the function $f$ on the set $\Omega$ it is necessary that

$$
\bigcup_{C \in E^{*}(u)}\left[-K^{+}(C)\right] \subset \bigcap_{C \in E^{*}(f)} \operatorname{cl}\left\{\mathbb{R}^{n} \backslash\left(-K^{+}(C)\right)\right\},
$$

where $K(C)=$ cone $\{C\}$ is the conic hull of the set $C, K^{+}(C)$ is the conjugate cone of $K(C)$.

Proof. As in the proof of the previous theorem it can be shown that if $x_{*}$ is a local minimum of the function $f$ on the set $\Omega$, then

$$
\min _{C \in E^{*}(f)} \max _{v \in C}\langle v, g\rangle \geq 0
$$

holds for any $g$ such that

$$
\min _{C \in E^{*}(u)} \max _{v \in C}\langle v, g\rangle \leq 0 .
$$

Therefore for any $g \in \mathbb{R}^{n}$ such that

$$
\exists \widetilde{C} \in E^{*}(u): \max _{v \in \widetilde{C}}\langle v, g\rangle \leq 0
$$

holds the condition

$$
\max _{v \in C}\langle v, g\rangle \geq 0 \quad \forall C \in E^{*}(f) .
$$

Inequality (15) is equivalent to the fact that there exists $\widetilde{C} \in E^{*}(u)$ such that $\langle v, g\rangle \leq 0$ for all $v \in \widetilde{C}$. Consequently there exists $\widetilde{C} \in E^{*}(u)$ such that $g \in$ $-K^{+}(\widetilde{C})$, where $K^{+}(\widetilde{C})$ is a conjugate cone of cone $\{\widetilde{C}\}$. Whence we conclude that $g \in \bigcup_{C \in E^{*}(u)}\left[-K^{+}(C)\right]$ is equivalent to condition (15). Therefore (15) and (16) implies (14). 
Corollary 8.1. Condition (14) can be interpreted as follows: for any hyperplane passing through the origin which nonpositive half-space contains at least one set $C \in E^{*}(u)$, there exists an element from $\widetilde{C}$ which lies in the nonnegative halfspace of this hyperplane for all $\widetilde{C} \in E^{*}(f)$.

Remark 3.1. If $x_{*}$ is an unconstrained local minimum of $f$ then condition (5) holds. Therefore

$$
\bigcap_{C \in E^{*}(f)} \operatorname{cl}\left\{\mathbb{R}^{n} \backslash\left(-K^{+}(C)\right)\right\}=\mathbb{R}^{n},
$$

which implies that inclusions (10) and (14) are always satisfied.

Now proceed to the minimum conditions in terms of (adjoint) lower exhauster of the function $f$.

Theorem 9. Let the regularity condition holds at the point $x_{*} \in \Omega$, families of sets $E_{*}(f)$ and $E_{*}(u)$ be lower exhausters in the sense of Hadamard of the functions $f$ and $u$ at the point $x_{*}$ respectively. Then for the point $x_{*}$ to be a local minimum of the function $f$ on the set $\Omega$ it is necessary that

$$
\bigcap_{C \in E_{*}(u)} \operatorname{cl}\left\{\mathbb{R}^{n} \backslash K^{+}(C)\right\} \subset \bigcup_{C \in E_{*}(f)} K^{+}(C),
$$

where $K(C)=$ cone $\{C\}$ is the conic hull of the set $C, K^{+}(C)$ is the conjugate cone of $K(C)$.

Proof. As in the proof of Theorem 7 it can be shown that if $x_{*}$ is a local minimum of the function $f$ on the set $\Omega$, then

$$
\max _{C \in E_{*}(f)} \min _{v \in C}\langle v, g\rangle \geq 0
$$

holds for any $g$ such that

$$
\max _{C \in E_{*}(u)} \min _{v \in C}\langle v, g\rangle \leq 0
$$

Therefore for any $g \in \mathbb{R}^{n}$ such that

$$
\min _{v \in C}\langle v, g\rangle \leq 0 \quad \forall C \in E_{*}(u)
$$

holds the condition

$$
\exists \widehat{C} \in E_{*}(f): \min _{v \in \widehat{C}}\langle v, g\rangle \geq 0 .
$$

Inequality (19) is equivalent to the fact that $g \in K^{+}(\widehat{C})$, where $K^{+}(\widehat{C})$ is a conjugate cone of cone $(\widehat{C})$. Thus (18) and (19) implies (17).

Corollary 9.1. Condition (17) can be interpreted as follows: for any hyperplane passing through the origin which nonpositive half-space contains an element from $C$ for all $C \in E_{*}(u)$, there exists at least one set $C \in E_{*}(f)$ which fully lies in the nonnegative half-space of this hyperplane.

Similarly can be proved the following result. 
Theorem 10. Let the regularity condition holds at the point $x_{*} \in \Omega$, families of sets $E_{*}(f)$ and $E^{*}(u)$ be a lower and an upper exhausters in the sense of Hadamard of the functions $f$ and $u$ at the point $x_{*}$ respectively. Then for the point $x_{*}$ to be a local minimum of the function $f$ on the set $\Omega$ it is necessary that

$$
\bigcup_{C \in E^{*}(u)}\left[-K^{+}(C)\right] \subset \bigcup_{C \in E_{*}(f)} K^{+}(C),
$$

where $K(C)=$ cone $\{C\}$ is the conic hull of the set $C, K^{+}(C)$ is the conjugate cone of $K(C)$.

Corollary 10.1. Condition (20) can be interpreted as follows: for any hyperplane passing through the origin which nonpositive half-space contains at least one set $C \in E^{*}(u)$, there exists at least one set $C \in E_{*}(f)$ which fully lies in the nonnegative half-space of this hyperplane.

Remark 3.2. If $x_{*}$ is an unconstrained local minimum of $f$ then condition (6) holds. Therefore

$$
\bigcup_{C \in E_{*}(f)} K^{+}(C)=\mathbb{R}^{n}
$$

which implies that inclusions (17) and (20) are always satisfied.

Analogously via Theorem 6 we can state and prove conditions for the maximum.

Theorem 11. Let the regularity condition holds at the point $x^{*} \in \Omega$, families of sets $E_{*}(f)$ and $E_{*}(u)$ be lower exhausters in the sense of Hadamard of the functions $f$ and $u$ at the point $x^{*}$ respectively. Then for the point $x^{*}$ to be a local maximum of the function $f$ on the set $\Omega$ it is necessary that

$$
\bigcap_{C \in E_{*}(u)} \operatorname{cl}\left\{\mathbb{R}^{n} \backslash K^{+}(C)\right\} \subset \bigcap_{C \in E_{*}(f)} \operatorname{cl}\left\{\mathbb{R}^{n} \backslash K^{+}(C)\right\},
$$

where $K(C)=\operatorname{cone}\{C\}$ is the conic hull of the set $C, K^{+}(C)$ is the conjugate cone of $K(C)$.

Theorem 12. Let the regularity condition holds at the point $x^{*} \in \Omega$, families of sets $E_{*}(f)$ and $E^{*}(u)$ be a lower and an upper exhausters in the sense of Hadamard of the functions $f$ and $u$ at the point $x^{*}$ respectively. Then for the point $x^{*}$ to be a local maximum of the function $f$ on the set $\Omega$ it is necessary that

$$
\bigcup_{C \in E^{*}(u)}\left[-K^{+}(C)\right] \subset \bigcap_{C \in E_{*}(f)} \operatorname{cl}\left\{\mathbb{R}^{n} \backslash K^{+}(C)\right\},
$$

where $K(C)=$ cone $\{C\}$ is the conic hull of the set $C, K^{+}(C)$ is the conjugate cone of $K(C)$.

Theorem 13. Let the regularity condition holds at the point $x^{*} \in \Omega$, families of sets $E^{*}(f)$ and $E_{*}(u)$ be an upper and a lower exhausters in the sense of Hadamard of the functions $f$ and $u$ at the point $x^{*}$ respectively. Then for the point $x^{*}$ to be a local maximum of the function $f$ on the set $\Omega$ it is necessary that

$$
\bigcap_{C \in E_{*}(u)} \operatorname{cl}\left\{\mathbb{R}^{n} \backslash K^{+}(C)\right\} \subset \bigcup_{C \in E^{*}(f)}\left[-K^{+}(C)\right],
$$


where $K(C)=$ cone $\{C\}$ is the conic hull of the set $C, K^{+}(C)$ is the conjugate cone of $K(C)$.

Theorem 14. Let the regularity condition holds at the point $x^{*} \in \Omega$, families of sets $E^{*}(f)$ and $E^{*}(u)$ be upper exhausters in the sense of Hadamard of the functions $f$ and $u$ at the point $x^{*}$ respectively. Then for the point $x^{*}$ to be a local maximum of the function $f$ on the set $\Omega$ it is necessary that

$$
\bigcup_{C \in E^{*}(u)}\left[-K^{+}(C)\right] \subset \bigcup_{C \in E^{*}(f)}\left[-K^{+}(C)\right],
$$

where $K(C)=$ cone $\{C\}$ is the conic hull of the set $C, K^{+}(C)$ is the conjugate cone of $K(C)$.

\section{An illustrative example}

Consider a function $f(x)=\left|x_{1}\right|-\left|x_{2}\right|$ on a set $\Omega=\left\{x \in \mathbb{R}^{2} \mid u(x) \leq 0\right\}$ at a point $x_{*}=(0,0)$, where

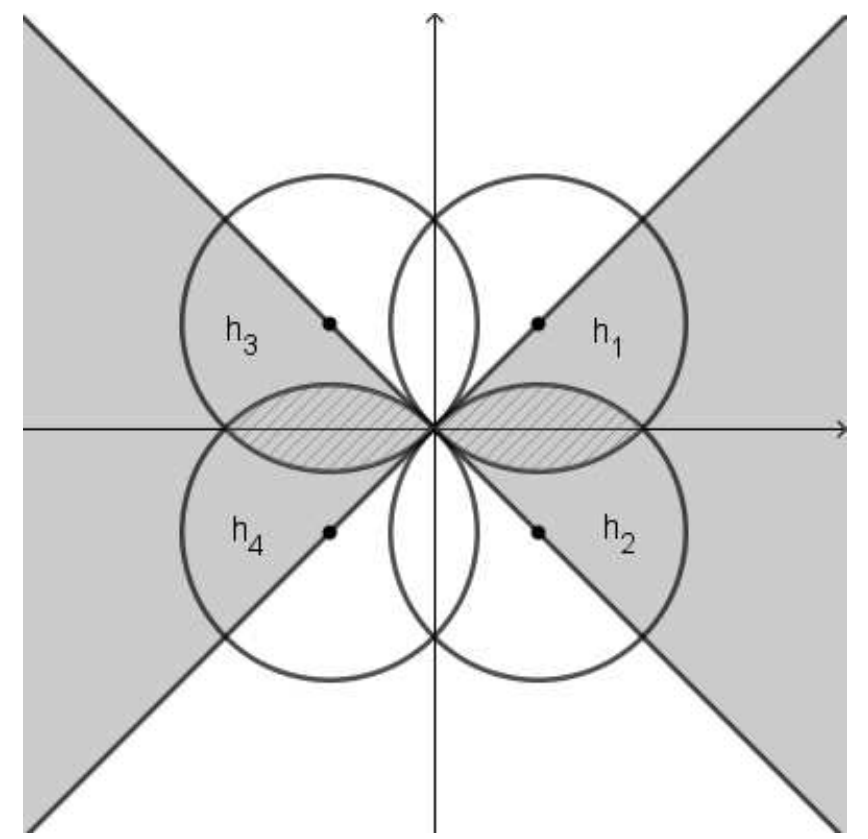

Figure 1: The set $\Omega$ (hatched area) and the cone $K_{a d}\left(x_{*}\right)$ (shaded area).

$$
\begin{aligned}
u(x) & =\min \left\{\max \left\{h_{1}(x), h_{2}(x)\right\}, \max \left\{h_{3}(x), h_{4}(x)\right\}\right\}= \\
& =\max \left\{\min \left\{h_{1}(x), h_{3}(x)\right\}, \max \left\{h_{2}(x), h_{4}(x)\right\}\right\},
\end{aligned}
$$

$\begin{array}{ll}h_{1}(x)=\frac{1}{2}\left[\left(x_{1}-1\right)^{2}+\left(x_{2}-1\right)^{2}\right]-1, & h_{2}(x)=\frac{1}{2}\left[\left(x_{1}-1\right)^{2}+\left(x_{2}+1\right)^{2}\right]-1, \\ h_{3}(x)=\frac{1}{2}\left[\left(x_{1}+1\right)^{2}+\left(x_{2}-1\right)^{2}\right]-1, & h_{4}(x)=\frac{1}{2}\left[\left(x_{1}+1\right)^{2}+\left(x_{2}+1\right)^{2}\right]-1 .\end{array}$ 
The functions $f$ and $u$ are Dini-directionally differentiable at the point $x_{*}$ and Lipschitz, therefore they are directionally differentiable in the sense of Hadamard [8]. It is also obvious that regularity condition holds at the point $x_{*}$.

Denote

$$
\begin{array}{ll}
C_{1}=\operatorname{co}\{(1,1) ;(-1,1)\}, & C_{2}=\operatorname{co}\{(1,-1) ;(-1,-1)\}, \\
C_{3}=\operatorname{co}\{(1,1) ;(1,-1)\}, & C_{4}=\operatorname{co}\{(-1,1) ;(-1,-1)\} .
\end{array}
$$

Then the following families are exhausters of the functions $f$ and $u$ at the point $x_{*}$ :

$$
E^{*}(f)=E_{*}(u)=\left\{C_{1}, C_{2}\right\}, \quad E_{*}(f)=E^{*}(u)=\left\{C_{3}, C_{4}\right\}
$$

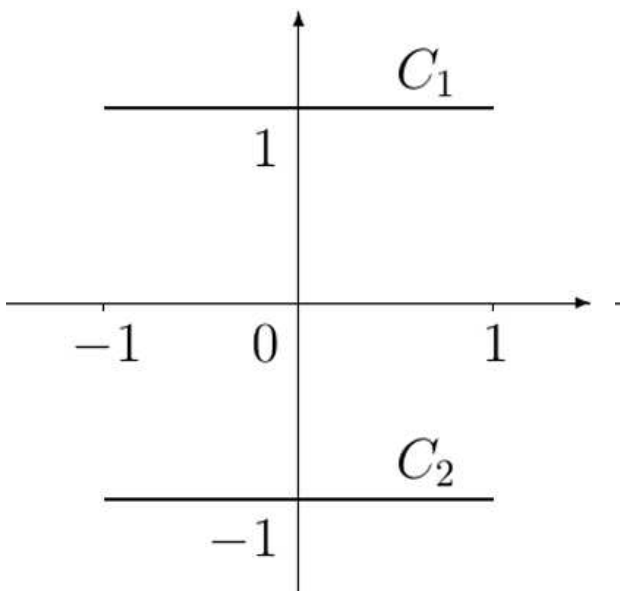

a

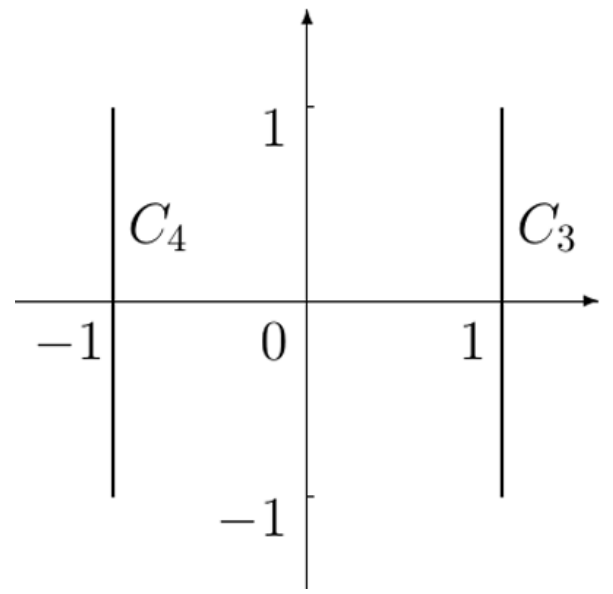

$\mathrm{b}$

Figure 2: Sets that form exhausters of the functions $f$ and $u$ at the point $x_{0} *$.

First check minimality conditions in terms of proper exhauster. Condition (10) from Theorem 7 is satisfied since

$$
\bigcap_{C \in E_{*}(u)} \operatorname{cl}\left\{\mathbb{R}^{n} \backslash K^{+}(C)\right\}=\bigcap_{C \in E^{*}(f)} \operatorname{cl}\left\{\mathbb{R}^{n} \backslash\left(-K^{+}(C)\right)\right\}=K\left(C_{3}\right) \bigcup K\left(C_{4}\right) .
$$

Condition (14) from Theorem 8 also holds:

$$
\bigcup_{C \in E^{*}(u)}\left[-K^{+}(C)\right]=\bigcap_{C \in E^{*}(f)} \operatorname{cl}\left\{\mathbb{R}^{n} \backslash\left(-K^{+}(C)\right)\right\}=K\left(C_{3}\right) \bigcup K\left(C_{4}\right) .
$$

Now pass to the minimality conditions in terms of adjoint exhauster.

We have

$$
\bigcap_{C \in E_{*}(u)} \mathrm{cl}\left\{\mathbb{R}^{n} \backslash K^{+}(C)\right\}=\bigcup_{C \in E_{*}(f)} K^{+}(C)=K\left(C_{3}\right) \bigcup K\left(C_{4}\right),
$$

therefore condition (17) from Theorem 9 is fulfilled.

Condition (20) from Theorem 10 also holds:

$$
\bigcup_{C \in E^{*}(u)}\left[-K^{+}(C)\right]=\bigcup_{C \in E_{*}(f)} K^{+}(C)=K\left(C_{3}\right) \bigcup K\left(C_{4}\right) .
$$




\section{Conclusion}

We derived new optimality conditions for an exhausterable function on an exhausterable set and showed how they can be applied to practical problems.

It should be noted that constrained optimality conditions for an exhausterable function on an abstractive theoretical cone were provided in [15]. But these results were only the first step, as one of the most important problem of constructing such a cone in specific cases remained open. In the present paper we considered the case when a feasible set is given via an exhausterable function, described Bouligand cone in terms of exhausters of this function and therefore got optimality conditions in terms of these exhausters. Obtained results can be applied to a wide class of nondifferentiable optimization problems.

\section{Acknowledgements}

The reported study was supported by Russian Science Foundation, research project No. 18-71-00006.

\section{References}

[1] Rockafellar, R.T.: Convex Analysis, Princeton University Press, Princeton, N.J. (1970)

[2] Clarke, F.H.: Optimization and Nonsmooth Analysis WileyInterscience. New York (1983)

[3] Hare, W., Nutini, J.: A derivative-free approximate gradient sampling algorithm for finite minimax problems. Comput. Optim. Appl. 56, 1-38 (2013)

[4] Mordukhovich, B.S.: Metric approximations and necessary optimality conditions for general classes of extremal problems. Soviet Math. Dokl., 22, 526-530 (1980)

[5] Michel, P., Penot J.-P.: Calcul sous-differentiel pour les fonctions lipschitziennes et non lipschitziennes. C.R. Acad. Sci. Paris. 298, 269-272 (1984)

[6] Hiriart-Urruty, J.-B., Lewis, A.S.: The Clarke and Michel-Penot Subdifferentials of the Eigenvalues of a Symmetric Matrix. Comput. Optim. Appl. 13, $13-23$ (1999)

[7] Pshenichny, B.N.: Convex Analysis and Extremal Problems (in Russian). Nauka, Moscow (1980)

[8] Demyanov, V.F., Rubinov, A.M.: Constructive Nonsmooth Analysis. Approximation \& Optimization. Vol. 7. Peter Lang, Frankfurt am Main, iv+416 pp. (1995)

[9] Demyanov, V.F., Rubinov, A.M.: Exhaustive families of approximations revisited. In: From Convexity to Nonconvexity. Nonconvex Optim. Appl. Vol. 55, 43-50. Kluwer Academic, Dordrecht (2001) 
[10] Demyanov, V.F.: Exhausters af a positively homogeneous function. Optimization. 45, 13-29 (1999)

[11] Demyanov, V.F.: Exhausters and Convexificators - New Tools in Nonsmooth Analysis. In: V. Demyanov and A. Rubinov: (Eds.) Quasidifferentiability and related topics. Dordrecht: Kluwer Academic Publishers, 85-137 (2000)

[12] Demyanov, V.F., Roshchina, V.A.: Optimality conditions in terms of upper and lower exhausters. Optimization. 55, 525-540 (2006)

[13] Abbasov, M.E., Demyanov, V.F.: Extremum conditions for a nonsmooth function in terms of exhausters and coexhausters. Proceedings of the Steklov Institute of Mathematics. 269, 6-15 (2010)

[14] Abbasov, M.E., Demyanov, V.F.: Proper and adjoint exhausters in nonsmooth analysis: optimality conditions. J. Glob. Optim. 56, 569-585 (2013)

[15] Demyanov V.F., Roshchina V.A.: Constrained Optimality Conditions in Terms of Proper and Adjoint Exhausters. Appl. Comput. Math., 4, 144-124 (2005)

[16] Castellani, M.: A dual representation for proper positively homogeneous functions. J. Global Optim. 16, 393-400, (2000)

[17] Abbasov, M.E.: Comparison Between Quasidifferentials and Exhausters. J. Optim. Theory Appl., 175(1), 59-75 (2017)

[18] Demyanov, V.F.: Proper exhausters and coexhausters in nonsmooth analysis. Optimization. 61, 1347-1368 (2012)

[19] Roshchina V.A.: Reducing Exhausters. J. Optim. Theory Appl. 136, 261273 (2008)

[20] Luderer, B., Weigelt, J.: A solution method for a special class of nondifferentiable unconstrained optimization problems. Comput. Optim. Appl. 24, 83-93 (2003)

[21] Küçük, M., Urbanski, R., Grzybowski, J. et al.: Reduction of Weak Exhausters and Optimality Conditions via Reduced Weak Exhausters. J Optim Theory Appl. 165, 693-707 (2015)

[22] Abbasov, M.E.: Geometric conditions of reduction of exhausters. J. Glob. Optim. (2018). https://doi.org/10.1007/s10898-018-0683-5

[23] Abbasov, M.E.: Generalized exhausters: Existence, construction, optimality conditions. J. Glob. Optim. 11, 217-230 (2015)

[24] Murzabekova, G.E.: Implicit function theorem for nonsmooth systems by means of exhausters. J. Comput. Syst. Sci. Int. 48, 574-580 (2009) 